REVIEW

\title{
Differential diagnosis of common tremor syndromes
}

R Bhidayasiri

Postgrad Med J 2005;81:756-762. doi: 10.1136/pgmj.2005.032979

Tremor is one of the most common involuntary movement disorders seen in clinical practice. In addition to the detailed history, the differential diagnosis is mainly clinical based on the distinction at rest, postural and intention, activation condition, frequency, and topographical distribution. The causes of tremor are heterogeneous and it can present alone (for example, essential tremor) or as a part of a neurological syndrome (for example, multiple sclerosis). Essential tremor and the tremor of Parkinson's disease are the most common tremors encountered in clinical practice. This article focuses on a practical approach to these different forms of tremor and how to distinguish them clinically. Evidence supporting various strategies used in the differentiation is then presented, followed by a review of formal guidelines or recommendations when they exist.

\section{Correspondence to: Dr R Bhidayasiri, Department of Neurology, Reed Neurological Research Institute, UCLA Medical Center, 710 Westwood Plaza, Los Angeles, CA 90095, USA; rbh@ucla.edu}

Submitted 24 January 2005 Accepted 16 April 2005
$\mathrm{T}$ remor is one of the most common involuntary movement disorders seen in clinical practice. It is defined as an involuntary, approximately rhythmic, and roughly sinusoidal movement of one or more body parts. It is differentiated from other involuntary movement disorders, such as chorea, athetosis, ballism, tics, and myoclonus, by its repetitive, stereotyped movements of a regular amplitude and frequency. Tremor may be confused with rhythmic myoclonus (incorrectly termed cortical tremor), which is typically characterised by brief muscle twitches, confined to one limb or to adjacent body regions, associated with spike-wave complexes on the electroencephalogram (EEG) or spinal lesions. Clonus, unlike tremor, represents a rhythmic movement, which is increased by muscle stretching. Asterixis can be distinguished from tremor on the basis of electromyographic (EMG) findings of prolonged absence of EMG activity during "flapping" or abduction of the upper extremities. Stereotypies may have rhythmic components, but nevertheless are dominanted by complex movements. Lastly, epilepsia partialis continua (EPC) can produce regular jerks of the arm or hand, which can be difficult to distinguish from tremor. EPC is associated with EEG changes (which may need to be identified with back-averaging techniques), and MRI changes in contralateral sensorimotor cortex.

The first step in evaluating any patient with tremor is to characterise the tremor. Various types of tremor can be distinguished clinically, based on the activation condition, frequency, and topographical distribution. Different classifications of tremor have been proposed although the most useful and widely accepted classification divides tremor according to the behaviour it occurs, that is rest and action tremor, which is further subdivided into postural and kinetic tremor (table 1). ${ }^{1-3}$ Action tremor, the most prevalent of these types of tremor, occurs during sustained extension of the arm and during voluntary movements, such as writing or typing. Resting tremor is suspected, if it occurs with the patient sitting with his arms firmly supported without any voluntary activities, if it increases with mental stress (counting backwards), and if it is suppressed by voluntary movements. The most common cause of resting tremor is idiopathic Parkinson's disease (PD). The most common cause of postural and kinetic tremor is essential tremor (ET). Physiological tremor is an action tremor and is present in every healthy person under certain conditions. Tremor can present alone or as part of a neurological syndrome, for example multiple sclerosis, dystonia, and neuropathy. This article discusses different types of tremor with an emphasis on salient features and how to distinguish them clinically. Evidence supporting various available strategies is then presented, followed by a review of established guidelines.

\section{ESSENTIAL TREMOR: THE MOST COMMON FORM OF ACTION TREMOR}

Action tremor refers to any tremor that is produced by voluntary contraction of muscles, including postural, isometric, and kinetic tremor. The last includes intention tremor. As there are no validated serological, radiological, and pathological markers in ET, the diagnosis is primarily based on clinical findings (box 1). ${ }^{2}$ Therefore, the examination should be comprehensive. Firstly, observe the patient sitting at rest to note whether there is evidence of a resting tremor of the head, hands, or legs. Then, ask the patient to stretch out the arms and hands completely and look for a postural tremor, followed by checking fingernose-finger movements looking for a kinetic tremor. Typically, essential tremor is an action tremor, either postural or kinetic in character, mainly affecting the hands. It is usually bilateral with a frequency of $4 \mathrm{~Hz}$ to $12 \mathrm{~Hz}$ and largely symmetrical. ${ }^{4}$ The upper limbs are affected in about $95 \%$ of patients, followed by head (34\%),

\footnotetext{
Abbreviations: EMG, electromyography; EEG, electroencephalography; EPC, epilepsia partialis continua; PD, Parkinson's disease; ET, essential tremor; PET, positron emission tomography; DAT, dopamine transporter; DBS, deep brain stimulation; VIM, ventral intermedius nucleus
} 
Table 1 Classification of tremor

\begin{tabular}{ll}
\hline Type of tremor & Definition \\
\hline Rest tremor & $\begin{array}{l}\text { Tremor that occurs in a body part that } \\
\text { is not voluntarily activated and is } \\
\text { completed supported against gravity. }\end{array}$ \\
Any tremor that is produced by \\
voluntary contraction of muscle, \\
including postural, isometric, and \\
kinetic tremor. The last includes \\
intention tremor. \\
Tremor that is present while \\
voluntarily maintaining a position \\
against gravity. \\
Tremor that occurs during any \\
voluntary movement. It may include \\
visually or non-visually guided \\
movements. Tremor during target \\
directed movement is called intention \\
tremor. \\
Tremor that occurs as a result of \\
muscle contraction against a rigid \\
stationary object. \\
Kinetic tremor that may appear or \\
become exacerbated during specific \\
activities.
\end{tabular}

lower limbs (20\%), voice (12\%), face and trunk (5\%). ${ }^{2}$ With the passage of time, the frequency of the tremor decreases and the amplitude may increase. ${ }^{5}$ The prevalence ranges from $0.4 \%$ to $6.7 \%$ in persons over 40 years old so it is the most common type of tremor. ${ }^{6-8}$ Many studies have shown that ET is much more prevalent than tremor of PD (up to 20 times difference). ${ }^{9}{ }^{10}$ However, some experts suspected that the condition might be overdiagnosed. ${ }^{11}$ Although the condition is both clinically and genetically heterogeneous, half of the cases are considered familial with an autosomal dominant pattern of inheritance. ${ }^{912-14}$ Two different chromosomal regions have been linked to familial ET, one on chromosome $3 \mathrm{q} 13^{15}$ and another on chromosome $2 \mathrm{p} 22-25 .{ }^{16}$ However, no specific gene mutations have been identified to date. The penetrance is thought to be high, suggesting that $89 \%$ of patients at risk have signs of ET by the age of $65 .{ }^{6}{ }^{17}$ The age of onset is typically $60-70$ years, but not uncommonly before 60 years, and both sexes are equally affected. The tremor commonly involves the head, jaw, neck, facial muscles, tongue, and upper extremities but not the lip, which suggests the tremor of PD in those cases.

Clinically, the differentiation between ET and tremor of PD can be difficult (table 2). However, important features that support the tremor to be parkinsonian in origin include asymmetric onset and it being at rest although $40 \%$ of tremor in PD can be of mixed type of postural and resting tremors. Tremor that occurs during walking usually suggests an underlying diagnosis of PD. In addition, patients with ET typically lack prominent extrapyramidal signs, including bradykinesia, manifesting as progressive decrement in amplitude and speed with "re-setting", postural instability or rigidity. Fifty per cent of ET patients are alcohol responsive but only temporarily. ${ }^{18}$ Sometimes, it can be difficult to determine if bradykinesia is present in a patient with pronounced postural tremor. In these circumstances, other factors such as the presence of hypomimia and generalised bradykinesia may need to be taken into account. A "no-no" or "yes-yes" head tremor is characteristic of ET and occurs only rarely in PD. Handwriting is usually small and illegible in PD but large and tremulous in ET (fig 1). If a noticeable tremor is noted with speaking, ET is probable in most cases with a possibility of isolated voice tremor in a minority. Most patients with ET do not have abnormal neurological findings

\section{Box 1 Clinical criteria for essential tremor ${ }^{2}$}

\section{Definite essential tremor}

- Postural tremor of moderate amplitude is present in at least one arm

- Tremor of moderate amplitude is present in at least one arm during at least four tasks, such as pouring water, using a spoon to drink water drinking water, finger-tonose manoeuvre, and drawing a spiral.

- Tremor must interfere with at least one activity of daily living.

- Medications, hypothyroidism, alcohol, and other neurological conditions are not the cause of tremor.

\section{Probable essential tremor}

- Tremor of moderate amplitude is present in at least one arm during at least four tasks, or head tremor is present.

- Medications, hyperthyroidism, alcohol, and other neurological conditions are not the cause of tremor.

except the tremor although signs of mild cerebellar dysfunction can be seen, supported by a recent study using positron emission tomography (PET) showing increased cerebellar activation. ${ }^{19}$ When the presentation is atypical, functional brain imaging with positron emission tomography and the radiotracer 18-fluorodopa (FDOPA-PET) may permit the diagnosis of PD in early stage, recording and quantifying the deficiency of dopamine synthesis and storage within presynaptic striatal nerve terminals. In addition, dopamine transporter (DAT) single photon emission computed tomography (SPECT), such as ${ }^{123}$ I-ß-FP-CIT, can effectively distinguish between ET and PD in an early stage of the disease with the results being within normal limits in ET. ${ }^{21}$ Apart from excluding the possibility of early PD, a careful drug history is mandatory as many drugs are capable of producing postural and kinetic tremors (box 2). These drugs include $\beta$-adrenergic agonists, valproic acid, thyroxin, tricyclic antidepressants, selective serotonin reuptake inhibitors (SSRIs), and lithium. These drugs may cause increased physiological tremor that may be difficult to distinguish it from ET. Therefore, clinicians must maintain a high level of suspicion when a tremor develops after the start of a drug treatment. The possibility of Wilson's disease should always be considered in any patient with an action tremor who is younger than 40 years of age. A low serum ceruloplasmin is useful screening test although not diagnostic and the level of less than $200 \mathrm{mg} / \mathrm{l}$ has $95 \%$ sensitivity for this condition. A slit-lamp examination for Kayser-Fleischer ring should also be considered. However, patients with Wilson's disease usually present with dysarthria, dystonia, and parkinsonism and very rarely present with isolated action tremor. ${ }^{22}$

ET is believed to be of a central nervous system origin, but a reproducible neuropathology has not been described. A central aetiology was partly supported by the beneficial effect of thalamotomy, thalamic deep brain stimulation (DBS), and drugs that act centrally. Numerous experimental physiological and functional imaging studies have also implicated dysfunction in brain stem structures, including the inferior olive, locus coeruleus, red nucleus, thalamus, but cerebellum seems to be a prime candidate for the site of dysfunction in ET. ${ }^{23-25}$ It is probable that ET occur as a result of an abnormal oscillator of a CNS "pacemaker" in a currently unknown exact location that can be increased or suppressed by reflex pathways. 
Table 2 Features differentiating tremor of PD from $\mathrm{ET}^{72}$

\begin{tabular}{lll}
\hline Features & Parkinson's tremor & Essential tremor \\
\hline Tremor & $\begin{array}{l}\text { At rest, increases with walking. } \\
\text { Decreases with posture holding or } \\
\text { action }\end{array}$ & Posture holding or action \\
Frequency & $3-6 \mathrm{~Hz}$ & $5-12 \mathrm{~Hz}$ \\
Distribution & Asymmetrical & Symmetrical (mostly) \\
Body parts & Hands and legs & Hands, head, voice \\
Writing & Micrographia & Tremulous \\
Course & Progressive & Stable or slowly progressive \\
Family history & Less common (1\%) & Often (30\%-50\%) \\
Other neurological signs & Bradykinesia, rigidity, loss of & None \\
pubstances that improve tremor & $\begin{array}{l}\text { Levodopa, anticholinergics } \\
\text { Surgical treatment }\end{array}$ & $\begin{array}{l}\text { Patcohol, propranolol, primidone } \\
\text { parkinsonian features, requiring } \\
\text { subthalamic nucleus or internal globus } \\
\text { pallidus deep brain stimulation (DBS) }\end{array}$ \\
\hline
\end{tabular}

Unfortunately, pharmacological treatment of ET remains unsatisfactory. Probably, ET is not as benign as it is often referred as benign essential or familial tremor. About 15\%$25 \%$ of patients with ET retire prematurely and $60 \%$ of patients choose not to apply for a job or promotion because of the uncontrollable shaking of their hands. ${ }^{4}$ The two most often used drugs are non-selective $\beta$ blockers (for example, propranolol) and primidone. Because these drugs can result in multiple side effects, especially during the titration phase, they are not recommended for mild cases that do not cause dysfunction or social embarrassment. Tremor of different body parts and various tremor subtypes may also have different pharmacological responsiveness. In general, the start of specific pharmacological treatments is typically based on patient age, coexistent conditions, prior exposure to drug therapy, concurrent drug therapies, contraindications, physician and patient bias, as well as benefits and potential adverse effects of certain agents. Drug dose is initially low, gradually titrated upward as tolerated, and adjusted as appropriate to identify the most efficacious dose with a minimum of adverse effects (regulation of dose). If the drug is of no benefit at a dose that causes adverse effects, dose levels are gradually tapered down and treatment is eventually stopped. If a drug is reported to be beneficial, it may be continued at the regulated doses and the next drug may be added to the drug regimen. If the response to a drug is adequate and the dose well tolerated, the physician may keep the patient at the same dose or decide to increase the dose level (and continue to monitor tolerance). Propranolol, a non-selective antagonist, is more effective than selective $\beta_{1}$ activity, with the dose of at least $120 \mathrm{mg}$ /day resulting in a significant reduction in the severity of tremor. ${ }^{26}{ }^{27}$ In a dose response study of propranolol, $240-320 \mathrm{mg}$ /day was found to be the optimal dose range. ${ }^{28}$ Furthermore, long acting propranolol (propranolol LA) has been shown to be equally effective as conventional propranolol and has better compliance. ${ }^{29}$ Propranolol is generally well tolerated. However, relative contraindications, including asthma, heart failure, arterioventricular block, and diabetes mellitus, have limited its use in some patients. The mechanism of propranolol in ET is not exactly known although central and peripheral mechanisms have been proposed. ${ }^{30} 31$ In general, 50\%-70\% of patients obtain symptomatic relief from propranolol, but dramatic improvement occurs in a much smaller percentage. Similarly, primidone, an anticonvulsant in doses of up to $750 \mathrm{mg} /$ day, has been shown to be effective than placebo in reducing tremor. Although initial tolerability has limited the use of primidone, we find that slow titration, beginning as low as $12.5 \mathrm{mg} /$ day, may lessen side effects (mainly drowsiness) and increase tolerability. The mechanism of action of primidone's antitremor effect is also unknown. Phenobarbital is one of primidone's active metabolites but it has little, if

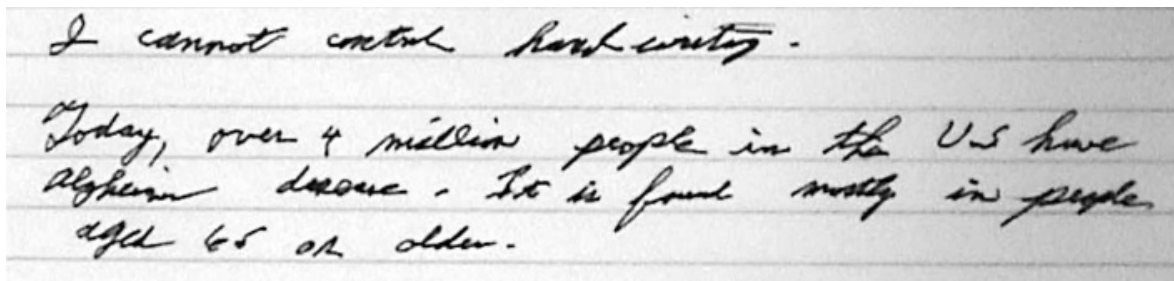

Figure 1 Comparison of handwriting in patients with $\mathrm{PD}$ (top) and $\mathrm{ET}$ (bottom). Note that the handwriting in PD is small and illegible in contrast with $\mathrm{ET}$, which is large and tremulous.

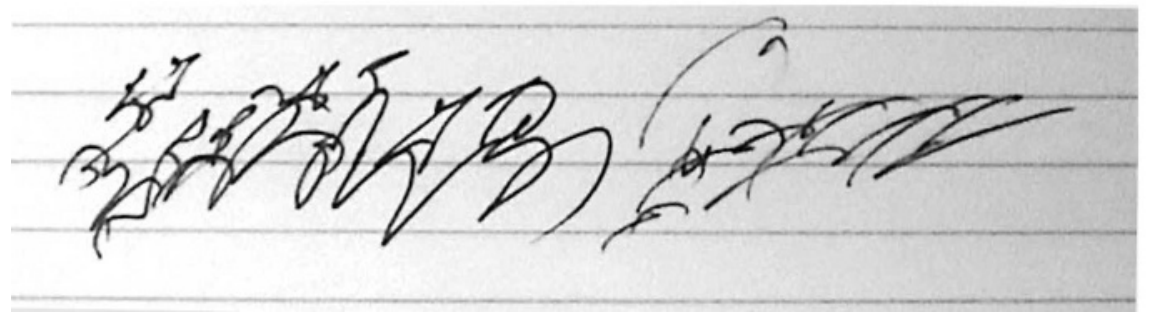


any, antitremor effect on its own. Common reported side effects include nausea, vertigo, drowsiness, and unsteadiness. Koller et $a^{32}$ showed in a placebo controlled study that primidone (50-1000 $\mathrm{mg} /$ day) significantly reduced the amplitude of hand tremor in both untreated and propranolol treated patients. There was no correlation between therapeutic response and serum concentrations. Neither drug was conclusively shown to be superior to the other but more patients had a preference for primidone than for propranolol in one study. ${ }^{33}$

In addition to the first line treatments, many other drugs have been used as monotherapy or adjunctive treatment. Topiramate has been shown to be effective as well as gabapentin and alprazolam. ${ }^{34}$ Theophylline, flunarizine, olanzapine have been used with variable success. Intramuscular injections of botulinum toxin type A into intrinsic hand muscles can be considered in medically resistant cases. ${ }^{36}$ Lastly, DBS in the ventral intermedius nucleus (VIM) of the thalamus in ET (Activa Tremor Control Therapy) is effective, with over $90 \%$ of patients having a satisfactory result. Because this rate of improvement cannot generally be achieved with current pharmacological therapy and several long term studies have also shown the long term efficacy, the Food and Drug Administration (FDA) has approved this device for medically intractable cases and it has now replaced thalamotomy. ${ }^{37} 38$

\section{PARKINSONIAN TREMOR: THE MOST COMMON FORM OF RESTING TREMOR}

The tremor in PD typically occurs at rest and becomes less prominent with voluntary movement. It typically occurs initially in the distal upper extremity, and over time, moves proximally and then to the other upper extremity, again in a distal to proximal pattern. Seventy per cent of patients with PD present with tremor and it usually has a better prognosis, compared with PD patients with early postural instability and akinesia. Action or postural tremor does occur in PD, either alone or in combination, making the diagnosis difficult in some cases, especially in the early stage. ${ }^{1}$ In fact, pure rest tremor is infrequent in PD; more common is the combination of rest and postural kinetic tremors. Isolated postural and kinetic tremor rarely occurs in PD. As a result of the variability of the clinical expression of tremors in $\mathrm{PD}$, the definition is based on the general diagnosis of PD rather than on specific features of tremors. Only the rest tremor component is by itself, a positive diagnostic criterion for PD

Box 2 Commonly used drugs that may cause tremor

- $\beta_{2}$ adrenergic agonists

- Valproic acid

- Lamotrigine

- Lithium

- Tricyclic antidepressants

- Antihistamines

- Thyroxine

- Amiodarone

- Nifedipine

- Neuroleptics

- Theophylline

- Nicotine

- Monoamine oxidase inhibitors

- Cyclosporin A but other tremors are often seen. When the diagnosis is unclear, levodopa trial may be considered to record the remarkable improvement in patients with $\mathrm{PD}$. In contrast with akinesia and rigidity, the response of parkinsonian tremor to dopaminergic treatment can be so variable and it is the overall improvement that counts and supports the diagnosis. ${ }^{39}$ A variety of agents have been used for tremor in $\mathrm{PD}$, including levodopa, dopamine agonists, anticholinergics, budipine, and as second line treatments, clozapine, propranolol, and clonazepam. ${ }^{40}$ However, double blind, randomised trials specifically assessing their efficacy in tremor in early PD are few with different methodologies and the results are variable. ${ }^{40-42}$ Anticholinergics, such as trihexylphenidyl, have been shown to be effective but rarely used now because of its side effects, especially in the elderly population. ${ }^{43}$ Therefore, anticholinergics are not generally recommended to patients with cognitive decline or elderly patients over 65 years of age. Both dopaminergic and anticholinergics are probably equally effective in parkinsonian tremor, but dopaminergic substances additionally improve other parkinsonian signs. Dopamine agonists, such as pramipexole and ropinirole, are probably the most effective antitremor drugs among all dopaminergic treatments and should be considered in all newly diagnosed tremor predominant PD patients who have no cognitive impairment. ${ }^{44-46}$ Improvement of tremor has also been reported with other dopamine agonists, including pergolide and bromocriptine. ${ }^{47} 48$ Dopamine agonists are also useful in advanced PD patients with tremor that is refractory to levodopa and anticholinergics. ${ }^{45}$ As for akinesia and rigidity, after longer disease duration the additional use of levodopa may become necessary for adequate control of resting tremor in many patients.

Some patients have a predominant postural tremor in addition to their rest tremor. This form is uncommon and has been considered to be a combination of an ET with PD although the relation between postural tremor that is phenomenologically similar to ET and PD has not been well defined. Further studies are needed to define the relation between ET and other tremors, including PD and other task specific tremors.

\section{WHAT IS THE PHYSIOLOGICAL TREMOR?}

Physiological tremor is seen in all normal people when muscles are activated. The tremor is typically postural and is thought to arise from the resonant oscillation of a limb as a result of mechanical factors affecting it. Because the physiological tremor has $8-12 \mathrm{~Hz}$, and $\alpha$ rhythm in the electroencephalogram has a similar frequency $(7-13 \mathrm{~Hz})$, a common central hypothesis was raised. ${ }^{49}$ Physiological tremor can be barely visible to the naked eye and does not interfere with activities of daily living. The frequency of physiological tremor is $<6 \mathrm{~Hz}$ before age 9 years, increasing to $12 \mathrm{~Hz}$ by the mid-teen years, and decreasing slightly above 60 years. The frequency usually decreases when large inertia loads are applied to the limb, as shown with accelerometry and electromyography. ${ }^{50}$ The amplitude is typically so low as to be virtually undetectable under normal circumstances. Increased physiological tremor is defined by the easy visibility of high frequency, postural tremor with no evidence of an underlying neurological disease. ${ }^{1}$ Furthermore, the cause is usually reversible. Certain conditions can exacerbate physiologic tremor, for example stress and anxiety before public performance. Indeed, some professional performers have learned to avoid this response by taking a $\beta$ blocker before the event. Fatigue because of lack of sleep and consuming a large amount of caffeine can be precipitating factors although one study did not find physiological tremor to be significantly increased by caffeine. ${ }^{51}$ Relaxation sessions have been shown 
to decrease tremor significantly. ${ }^{52}$ In general, no drugs are usually warranted. However, a small dose of propranolol can be useful in some people, for example ophthalmic surgeons, when fine coordinative movements are required. ${ }^{40}$ Other conditions that can augment physiological tremor include thyrotoxicosis, pheochromocytoma, hypoglycaemia, withdrawal from opioids or sedatives.

Tremor is a common side effect of many drugs (box 2 lists commonly used drugs that can cause tremor). Various drugs and toxins can cause all types of tremor known clinically although increased physiological tremor is most commonly seen. Tremor is the dose limiting side effect of the $\beta_{2}$ adrenergic agonists, salbutamol and terbutaline, used to treat obstructive airway diseases. Tremor is usually seen within a month of starting valproic acid treatment and is more evident when a dose is $>750 \mathrm{mg}$ /day although it can also occur when the dose is within therapeutic range. It is the most common tremorogenic drug among anticonvulsants, affecting up to $25 \%$ of patients. ${ }^{53}$ Intention tremor may occur in patients on lithium. The occurrence rate increases with increasing serum lithium levels and manifests almost $100 \%$ in patients with lithium toxicity. ${ }^{54}$ Tardive tremor, a rare disorder, represents a separate entity in which, by definition, is caused by exposure to a dopamine receptor blocking agent (DRBA) within six months of the onset of symptoms and persisting for at least one month after stopping the offending drug. ${ }^{55}$ It is usually static in nature but can occur at rest and on intentional movements, such as eating and writing. Tremor can also occur as a toxic reaction to marijuana, and 3,4-methylenedioxymethamphetamine or ecstasy. ${ }^{56}$

\section{CEREBELLAR TREMOR}

Classic cerebellar tremor is often termed as intention tremor. The tremor is typically of low frequency below $5 \mathrm{~Hz}$. It is characteristically kinetic in nature and has an added volitional component and particularly affects the head and the upper half of the body. Postural tremor may be present, but rest tremor is usually absent. When kinetic tremor occurs or worsens as the target is reached, it is referred to as terminal tremor. In rare occasions, cerebellar tremor also has a rest component in which case it would be described as Holmes' tremor. In cerebellar tremor, the oscillations are of variable amplitude and are perpendicular to the direction of movement. It is usually best elicited during the finger-nosefinger or heel-shin-heel tests. Furthermore, cerebellar tremor is often associated with dysmetria, dyssynergia, and hypotonia. Titubation is another tremor that is probably a result of abonormality of the cerebellum or its afferent/efferent pathways and is a slow frequency oscillation depending on postural innervation. Its rhythmicity is, at times, the only sign distinguishing it from ataxia of the trunk. Multiple sclerosis is a common cause of cerebellar tremor. Other causes include Friedreich's ataxia, spinocerebellar degeneration, and cerebellar infarction. Although no clear correlations between cerebellar lesions and tremor have been established, lesions in the superior cerebellar peduncle and dentate nucleus are the most common reported sites resulting in intention tremor. ${ }^{57} 58$

Unfortunately, there is no established pharmacological treatment for cerebellar tremor. The results of medical treatment are often less than satisfactory. However, as multiple neurotransmitters as well as feedback pathways, including brain stem, thalamus and cortical neurons, seem to be involved, several drugs have been tried, but with variable success, including odansetron (5- $\mathrm{HT}_{3}$ antagonist), isoniazid, physostigmine, carbamazepine, and clonazepam.5960 In refractory cases, chronic DBS of the VIM (or less commonly nucleus ventralis oralis posterior and zona incerta) may provide an alternative. ${ }^{61}{ }^{62}$ Although few studies used highly standardised quantitative outcome measures, and follow up periods were generally one year or less, the data suggested that chronic DBS of the VIM produced improved tremor control in multiple sclerosis ${ }^{61}$ However, complete cessation of tremor is generally not achieved. There were some reported cases in which tremor control decreased over time, and frequent reprogramming became necessary.

\section{PSYCHOGENIC TREMOR}

The criteria suggestive of psychogenic tremor are sudden onset but rarely a remitting course (box 3). The onset is mostly associated with a stressful life event. According to a modified Fahn's criteria for psychogenic dystonia, the diagnosis of psychogenic tremor is accepted with the followings; (1) the major causes of symptomatic tremors (such as medications, thyroid dysfunction, and hormonal or metabolic dysfunction) have been excluded, (2) essential and parkinsonian tremors are excluded on the basis of clinical criteria, (3) no evidence for any other neurological disorders is present, and (4) the patients had a period without tremor of at least two weeks during the observation period. ${ }^{63}$ The tremor of psychogenic in origin is usually a combination of resting and postural or intention tremors and most often involves both arms, followed by the head and then the legs. The tremor may be continuous or intermittent with fluctuating frequency and amplitude, but lacks the physiological pattern. As mentioned, the onset is usually abrupt (73\%) with maximal disability $(46 \%)$ at the onset that had static course in $46 \%$ and fluctuating course in $17 \% .{ }^{64}$ Although certain criteria are provided for the diagnosis of psychogenic tremor, the diagnosis can be obvious in patients with generalised shaking. ${ }^{6365}$ In these instances, the shaking usually stops during the examination as they are exhausting for patients. Differential diagnosis in this setting is limited, but includes orthostatic tremor, essential stance tremors, or the rare stance tremor of PD.

The examination, especially the two clinical signs, are very useful in this situation; the entrainment of tremor frequency

\section{Box 3 Clinical features suggestive of}

\section{psychogenic tremor ${ }^{7}$}

- Abrupt onset

- Static course

- Spontaneous remission

- Unclassified tremor (complex tremors)

- Clinical inconsistencies

- Changing tremor characteristics

- Unresponsive to antitremor drugs

- Tremor increases with attention, and lessens with distractibility

- Responsive to placebo

- Absence of other neurological signs

- Multiple somatisations

- Multiple underdiagnosed conditions

- Spontaneous remissions or cures of symptoms

- No evidence of disease by laboratory or radiological investigations

- Employed in allied health professionals

- Litigation or compensation pending

- Presence of secondary gain

- Presence of psychiatric disease

- Reported functional disturbances in the past 
and the coactivation sign. ${ }^{66}$ Entrainment entails requiring the patient to maintain a tapping rhythm in an uninvolved body part (finger or foot) at a different frequency than the suspicious tremor. A psychogenic tremor automatically changes to the frequency that is being enforced on the uninvolved hand or foot, because it is difficult to maintain two different volitional movement frequencies simultaneously in two different body parts. During passive movement of the involved limb, an increased tone can be palpated by the examiner. Once the increased tone disappears, the tremor also disappears (coactivation sign). Cogwheeling in the setting of PD and ET differs from the present coactivation sign as the first is present over the whole range of movement of a particular joint. In contrast, coactivation in psychogenic tremor resembles voluntary coactivation with overlying rhythmic trembling. In addition, coactivation can produce bizarre positioning of the hands when they are outstretched. The absence of finger tremor is also suggestive of psychogenic in origin. While physiological and pathological tremors show a decrease of tremor amplitude when postural with and without loading is compared, psychogenic tremor tends to show an increase of their tremor amplitudes during loading.

Commonly, patients with psychogenic tremor often undergo a large number of diagnosis and therapeutic procedures before the final diagnosis is established. A review of medical history in these patients usually shows multiple functional somatic or psychosomatic illnesses. Once the diagnosis is made, most patients continue to have a fluctuating or constant course, followed by improving and progressive periods suggesting the prognosis is far from benign. The therapeutic success is also variable, but the treatment approach should include various combinations of psychotherapy as well as drugs, such as mild anxiolytics and antidepressants. While pharmacological treatment in organic tremor may reduce amplitude, but does not change the tremor frequency, the effect of treatment in psychogenic tremor usually varies from total suppression of tremor, especially when associated with the suggestion of a "cure" to no benefit. ${ }^{26}$ Interestingly, most of successfully treated patients were young. ${ }^{63}$

\section{OTHER TYPES OF TREMOR}

In addition to the tremors described above, they are other forms of tremor that are less common and some of them have only been reported in a few case studies. Of these, dystonic tremor is worth mentioning as many patients with dystonia have tremor and it is sometimes difficult to distinguish dystonic tremors from static tremors associated with dystonia, which occur unspecifically in regions unaffected by dystonia. Dystonic tremor is mainly a postural and kinetic tremor in an extremity or body part affected by dystonia and is not usually seen during complete rest. ${ }^{1}$ It is now considered as a distinct entity from ET, as it is irregular, has a broad range of frequencies (mainly less than $7 \mathrm{~Hz}$ ), and remains localised. A typical example is tremulous spasmodic torticollis. The tremor tends to be localised, asymmetric, and irregular in amplitude and periodicity. Many patients with dystonic tremor use their own tricks (geste antagoniste or sensory tricks) to reduce the tremor amplitude. These together with the absence of attempts at suppressing the tremor by voluntary muscle contractions are a fairly reliable diagnostic sign. Head tremor is common in patients with cervical dystonia and treatment with botulinum toxin often results in significant improvement of tremor as well as dystonia.

Orthostatic tremor is a rare disorder of middle aged or elderly people that is characterised by unsteadiness on standing, secondary to $16 \mathrm{~Hz}$ tremor in the lower extremities.
Characteristically, the tremors remit on walking, but disappear when sitting or lying down. ${ }^{1}$ Patients prefer to stand on a wide base but walk normally and only a fine ripple of muscle activity is visible. Confirmation of the diagnosis can be obtained by EMG showing a $16 \mathrm{~Hz}$ pattern in the leg muscles with the patient standing. In terms of treatment, the drug most commonly used is clonazepam, followed by levodopa and then drugs used for ET. Overall, the treatment response seems to be unsatisfactory, but some success has been reported with gabapentin (300$2400 \mathrm{mg} /$ day) in a small placebo controlled, double blind, crossover trial. ${ }^{67}$

Holmes' tremor is a term, proposed by the Ad Hoc Scientific Committee on Movement Disorders, to refer to previously used midbrain tremor, rubral tremor, thalamic tremor, myorhythmia, and Benedikt's syndrome. ${ }^{1}$ It is a symptomatic tremor of predominantly proximal limbs of low frequency $(<4.5 \mathrm{~Hz})$ during postural in nature, worsening during movement and goal directed tasks. Like cerebellar tremor, Holmes' tremor is almost always attributable to lesions, reported in upper brain stem, thalamus, or cerebellum, interrupting pathways in the midbrain tegmentum (rubro-olivocerebellorubral loop, rubrospinal fibres, nigrostriatal fibres), and the serotonergic brain stem telencephalic fibres. ${ }^{68}$ Palatal tremor, previously termed palatal myoclonus, can be either symptomatic attributable to brain stem and/or cerebellar lesions or essential without any identified brain lesions. In symptomatic palatal tremor, inferior olivary pseudohypertrophy is seen and considered as a hallmark for the condition. ${ }^{69}{ }^{70}$ Sleep does not abolish the symptoms. In essential cases, patients usually have characteristic ear clicks (rhythmic movements of the tensor veli palatini muscle), which do not present in a symptomatic variety. Botulinum toxin injection into each tensor veli palatini has been reported to be of some benefits. ${ }^{71}$ Tremors, mostly postural and kinetic, can also develop in patients with some forms of peripheral neuropathy, particularly demyelinating neuropathies (especially dysgammaglobulinaemic neuropathies). The term "cortical tremor" is a misnomer as it is not a tremor but a specific form of rhythmic myoclonus. ${ }^{68}$ Distinguishing rhythmic myoclonus from tremor (particularly Holmes' tremor) can be difficult because the driving muscle contractions can be so brisk resulting in longer pauses between individual jerks.

Tremor is a common problem seen in clinical practice. Among all types of tremor, essential tremor is the most common cause. In most cases of tremor, there is no diagnostic laboratory test to confirm or exclude a particular type of tremor and the diagnosis heavily relies on physician's own observation and thorough clinical examination as well as clinical history. In persons younger than 40 years of age, the possibility of Wilson's disease should be excluded, as it is a treatable and reversible condition if recognised promptly. Differentiation between essential tremor and tremor of PD is particularly important as the management and prognosis in these two conditions are vastly different. Almost all drugs used to treat tremor should be titrated slowly as side effects and tolerability are the main issues of compliance. The treatment should be evidence based. We recommend that the first line treatments, if available, should be firstly attempted, followed by second line treatments that are supported by prospective clinical trials before finally choosing drugs from anecdotal evidence.

Funding: Roongroj Bhidayasiri is supported by Lilian Schorr Postdoctoral Fellowship of Parkinson's Disease Foundation (PDF).

Conflicts of interest: none. 


\section{REFERENCES}

1 Deuschl G, Bain P, Brin M. Consensus statement of the Movement Disorder Society on Tremor. Ad Hoc Scientific Committee. Mov Disord 1998;13/suppl 3):2-23.

2 Elble RJ. Diagnostic criteria for essential tremor and differential diagnosis. Neurology 2000:54:S2-6.

3 Grimes DA. Tremor-easily seen but difficult to describe and treat. Can J Neurol Sci 2003;30(suppl 1):S59-63.

4 Louis, ed. Clinical practice. Essential tremor. N Engl J Med 2001:345:887-91.

5 Elble RJ. Essential tremor frequency decreases with time. Neurology 2000;55:1547-51.

6 Brin MF, Koller W. Epidemiology and genetics of essential tremor. Mov Disord 1998;13(suppl 3):55-63.

7 Bharucha NE, Bharucha EP, Bharucha AE, et al. Prevalence of Parkinson's disease in the Parsi community of Bombay, India. Arch Neurol 1988; $45: 1321-3$

8 Haerer AF, Anderson DW, Schoenberg BS. Prevalence of essential tremor. Results from the Copiah County study. Arch Neurol 1982:39:750-1.

9 Louis ED, Ford B, Frucht S, et al. Risk of tremor and impairment from tremor in relatives of patients with essential tremor: a community-based family study. Ann Neurol 2001;49:761-9.

10 Bain PG, Findley $\mathrm{L}$, Thompson PD, et al. A study of hereditary essential tremor. Brain 1994:117:805-24.

11 Schrag A, Munchau A, Bhatia KP, et al. Essential tremor: an overdiagnosed condition? J Neurol 2000;247:955-9.

12 Lovis ED, Ford B, Barnes LF. Clinical subtypes of essential tremor. Arch Neurol 2000:57:1194-8.

13 Louis ED, Barnes LF, Ford B, et al. Ethnic differences in essential tremor. Arch Neurol 2000;57:723-7.

14 Jankovic J. Essential tremor: a heterogenous disorder. Mov Disord 2002;17:638-44

15 Gulcher JR, Jonsson P, Kong A, et al. Mapping of a familial essential tremor gene, FET1, to chromosome 3q13. Nat Genet 1997;17:84-7.

16 Higgins JJ, Pho LT, Nee LE. A gene (ETM) for essential tremor maps to chromosome 2p22-p25. Mov Disord 1997; 12:859-64.

17 Rautakorpi I, Takala J, Marttila RJ, et al. Essential tremor in a Finnish population. Acta Neurol Scand 1982;66:58-67.

18 Chouinard S, Lovis ED, Fahn S. Agreement among movement disorder specialists on the clinical diagnosis of essential tremor. Mov Disord 1997; 12:973-6.

19 Wills AJ, Jenkins IH, Thompson PD, et al. A positron emission tomography study of cerebral activation associated with essential and writing tremor. Arch Neurol 1995;52:299-305

20 Asenbaum S, Pirker W, Angelberger P, et al. [1231]beta-CIT and SPECT in essential tremor and Parkinson's disease. J Neural Transm 1998:105:1213-28.

21 Benamer TS, Patterson J, Grosset DG, et al. Accurate differentiation of parkinsonism and essential tremor using visual assessment of [1231]-FP-Cl SPECT imaging: the [1 23I]-FP-CIT study group. Mov Disord 2000;15:503-10.

22 Roberts EA, Schilsky ML. A practice guideline on Wilson disease. Hepatology 2003:37:1475-92.

23 Deuschl G, Elble RJ. The pathophysiology of essential tremor. Neurology 2000:54:S14-20.

24 Deuschl G, Wenzelburger R, Loffler K, et al. Essential tremor and cerebellar dysfunction clinical and kinematic analysis of intention tremor. Brain 2000; 123:1568-80.

25 Wilms H, Sievers J, Deuschl G. Animal models of tremor. Mov Disord 1999:14:557-71.

26 Larsen TA, Calne DB. Essential tremor. Clin Neuropharmacol 1983;6:185-206.

27 Dietrichson $\mathrm{P}$, Espen $\mathrm{E}$. Effects of timolol and atenolol on benign essential tremor: placebo-controlled studies based on quantitative tremor recording. J Neurol Neurosurg Psychiatry 1981:44:677-83.

28 Koller WC. Dose-response relationship of propranolol in the treatment of essential tremor. Arch Neurol 1986;43:42-3.

29 Cleeves L, Findley U. Propranolol and propranolol-LA in essential tremor: 0 double blind comparative study. J Neurol Neurosurg Psychiatry 1988:51:379-84

30 Young RR. Essential-familial tremor and other action tremors. Semin Neurol 1982;2:386-91.

31 Jefferson $D$, Jenner $P$, Marsden $C D$. beta-Adrenoreceptor antagonists in essential tremor. J Neurol Neurosurg Psychiatry 1979:42:904-9.

32 Koller WC, Royse VL. Efficacy of primidone in essential tremor. Neurology 1986;36:121-4.

33 Gorman WP, Cooper R, Pocock P, et al. A comparison of primidone, propranolol, and placebo in essential tremor, using quantitative analysis. $J$ Neurol Neurosurg Psychiatry 1986;49:64-8.

34 Connor GS. A double-blind placebo-controlled trial of topiramate treatment for essential tremor. Neurology 2002;59:132-4.

35 Ondo W, Hunter C, Vuong KD, et al. Gabapentin for essential tremor: a multiple-dose, double-blind, placebo-controlled trial. Mov Disord 2000;15:678-82.

36 Brin MF, Lyons KE, Doucette J, et al. A randomized, double masked, controlled trial of botulinum toxin type A in essential hand tremor. Neurology $2001 ; 56: 1523-8$.
37 Koller W, Pahwa R, Busenbark K, et al. High-frequency unilateral thalamic stimulation in the treatment of essential and parkinsonian tremor. Ann Neurol 1997:42:292-9

38 Limousin P, Speelman JD, Gielen F, et al. Multicentre European study of thalamic stimulation in parkinsonian and essential tremor. J Neurol Neurosurg Psychiatry 1999;66:289-96.

39 Koller WC, Hubble JP. Levodopa therapy in Parkinson's disease. Neurology 1990;40(suppl):40-7.

40 Wasielewski PG, Burns JM, Koller WC. Pharmacologic treatment of tremor. Mov Disord 1998;13(suppl 3):90-100.

41 Koller WC. Pharmacologic treatment of parkinsonian tremor. Arch Neurol 1986;43:126-7.

42 Hughes AJ, Lees AJ, Stern GM. Apomorphine in the diagnosis and treatment of parkinsonian tremor. Clin Neuropharmacol 1990:13:312-17.

43 Katzenschlager R, Sampaio C, Costa J, et al. Anticholinergics for symptomatic management of Parkinson's disease. Cochrane Library. Issue 2. Oxford: Update Software, 2003

44 Navan P, Findley $\sqcup$, Jeffs JA, et al. Double-blind, single-dose, cross-over study of the effects of pramipexole, pergolide, and placebo on rest tremor and UPDRS part III in Parkinson's disease. Mov Disord 2003;18:176-80.

45 Pogarell O, Gasser T, van Hilten JJ, et al. Pramipexole in patients with Parkinson's disease and marked drug resistant tremor: a randomised, double blind, placebo controlled multicentre study. J Neurol Neurosurg Psychiatry 2002;72:713-20.

46 Schrag A, Keens J, Warner J. Ropinirole for the treatment of tremor in early Parkinson's disease. Eur J Neurol 2002;9:253-7.

47 Korczyn AD, Brunt ER, Larsen JP, et al. A 3-year randomized trial of ropinirole and bromocriptine in early Parkinson's disease. The 053 Study Group. Neurology 1999;53:364-70.

48 van Laar T, Lledo A, Quail D, et al. An analysis of the improvement in activities of daily living (ADL) and motor score associated with pergolide monotherapy in the treatment of Parkinson's disease. Eur J Neurol 1999:6:133.

49 Pizzuti GP, Byford GH, Cifaldi S, et al. Finger tremor and the central nervous system. J Biomed Eng 1992;14:356-9.

50 Deuschl G, Raethjen J, Lindemann $M$, et al. The pathophysiology of tremor. Muscle Nerve 2001;24:716-35.

51 Koller W, Cone S, Herbster G. Caffeine and tremor. Neurology 1987; 37:169-72.

52 Comby B, Chevalier G, Bouchoucha M. A new method for the measurement of tremor at rest. Arch Int Physiol Biochim Biophys 1992;100:73-8.

53 Karas BJ, Wilder BJ, Hammond EJ, et al. Valproate tremors. Neurology 1982;32:428-32

54 Vestergaard P. Clinically important side effects of long-term lithium treatment: a review. Acta Psychiatr Scand Suppl 1983;305:1-36

55 Stacy M, Jankovic J. Tardive tremor. Mov Disord 1992;7:53-7.

56 Demirkiran M, Jankovic J, Dean JM. Ecstasy intoxication: an overlap between serotonin syndrome and neuroleptic malignant syndrome. Clin Neuropharmacol 1996; 19:157-64.

57 Finsterer J, Muellbacher W, Mamoli B. Yes/yes head tremor without appendicular tremor after bilateral cerebellar infarction. J Neurol $\mathrm{Sci}$ 1996:139:242-5.

58 Thach WT, Goodkin HP, Keating JG. The cerebellum and the adaptive coordination of movement. Annu Rev Neurosci 1992;15:403-42.

59 Sandyk R. Successful treatment of cerebellar tremor with clonazepam. Clin Pharm 1985:4:615-18.

60 Trelles L, Trelles JO, Castro C, et al. Successful treatment of two cases of intention tremor with clonazepam. Ann Neurol 1984;16:621.

61 Wishart HA, Roberts DW, Roth RM, et al. Chronic deep brain stimulation for the treatment of tremor in multiple sclerosis: review and case reports. J Neurol Neurosurg Psychiatry 2003:74:1392-7.

62 Nandi D, Aziz TZ. Deep brain stimulation in the management of neuropathic pain and multiple sclerosis tremor. I Clin Neurophysiol 2004;21:31-9.

63 Deuschl G, Koster B, Lucking CH, et al. Diagnostic and pathophysiological aspects of psychogenic tremors. Mov Disord 1998;13:294-302.

$64 \mathrm{Kim}$ YJ, Pakiam AS, Lang AE. Historical and clinical features of psychogenic tremor: a review of 70 cases. Can J Neurol Sci 1999;26:190-5.

65 Koller W, Lang A, Vetere-Overfield B, et al. Psychogenic tremors. Neurology 1989;39:1094-9.

66 Uddin MK, Rodnitzky RL. Tremor in children. Semin Pediatr Neurol 2003; 10:26-34

67 Onofri M, Thomas A, Paci C, et al. Gabapentin in orthostatic tremor: results of a double-blind crossover with placebo in four patients. Neurology 1998;51:880-2.

68 Samie MR, Selhorst JB, Koller WC. Post-traumatic midbrain tremors. Neurology 1990;40:62-6.

69 Deuschl G, Toro C, Valls-Sole J, et al. Symptomatic and essential palatal tremor. 1. Clinical, physiological and MRI analysis. Brain 1994;117:775-88.

70 Deuschl G, Toro C, Hallett M. Symptomatic and essential palatal tremor. 2. Differences of palatal movements. Mov Disord 1994;9:676-8.

71 Cho JW, Chu K, Jeon BS. Case of essential palatal tremor: atypical features and remarkable benefit from botulinum toxin injection. Mov Disord 2001;16:779-82.

72 Bhidayasiri R, Waters MF, Giza CC. Neurological differential diagnosis: a prioritized approach. Oxford: Blackwell, 2005

73 Manyam BV. Uncommon forms of tremor. In: Watts RL, Koller WC, eds. Movement disorders: neurologic principles and practice. 2nd ed. New York McGraw-Hill, 2004:459-80 